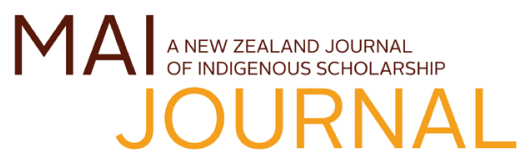

DOI: 10.20507/MAIJournal.2019.8.3.1

\title{
TE OROKOHANGA O TE AHO MATUA
}

\author{
Nā Kìmai Tocker*
}

\section{He whakarāpopoto}

Ko Te Aho Matua (2008) te tūāpapa o ngā kura kaupapa Māori o Aotearoa. Ko tā tēnei tuhinga, he tirotiro i ngā mahi a ngā manu tāiko hei whakatū i ngā kura kaupapa Māori.

Kei tēnei pepa hoki he whakamāramatanga e pā ana ki te take mō te tuhinga o Te Aho Matua. Ko tōna tikanga, he tautake Te Aho Matua e whakakotahi ana i ngā kura kaupapa Māori e whakaù ana ki te whakarauora i te reo Māori me te whakatairanga i ngā tikanga Māori. Kei Te Aho Matua ngā aratohu kaupapa here mā ngā whānau me ngā pouako hei whakahaere i te kura mō ngā tamariki tau 1-8.

He whakahokinga mahara tēnei tuhinga ki ngā whawhai a ngā kaihanga i whakapau kaha ki te whakatū i ngā kura kaupapa Māori ki Tāmaki Makaurau me te tuhi i Te Aho Matua. I te tīmatatanga, nō te whānau o te kōhanga reo rātou, ōtira i te ngoikoretanga o ngā kura auraki ki te whakawhanake tonu i te reo o ā rātou tamariki, i huri rātou ki te whakatū i à rātou ake kura. Kāore hoki i tautokohia e te kāwanatanga. Nā te kaha o ngā wero a ngā whānau nei, i tuohu mai te kāwanatanga ki ā rātou tono. Nā reira, i whakaturetia ngā kura kaupapa Māori e te kāwanatanga i te tau 1989, kia whiwhi pūtea ai rātou. Whai muri i tērā, i whakaturehia hoki Te Aho Matua i te tau 1999.

\section{He kupu matua}

kura kaupapa Māori, Te Aho Matua, te whakature o te kura kaupapa Māori me Te Aho Matua

\section{Te whakatakinga}

I te tau 1989, i tīmata taku tamāhine ki tētahi o ngā kura kaupapa Māori ki Tāmaki Makaurau. I runga i taku whakapono ki te kaupapa, i whakaae mai a Graham Smith rātou ko Linda Smith, ko Elizabeth Rata, ko Pita Sharples, ko Kāterina Te Heikōkō Mataira, ko Kathy Dewes ki te kōrero ki ahau mō taku rangahau tohu kairangi (Tocker, 2014) e pā ana ki te tīmatanga o te kura kaupapa Māori me te orokohanga o Te Aho Matua. Ko ngā mātanga nei ētahi o rātou i para i te huarahi kia whakarauoratia ai tō tātou reo rangatira. He kaha nō rātou ki te whawhai ki te kāwanatanga i waenganui i ngā tau 1980-1990 ki te whakatū i tētahi kura reo Māori kia ora ai te reo o à rātou tamariki kātahi anō ka puta i te kōhanga reo.

Ko te pūtake o taku tuhinga ko te ao Māori. Nā te whai i ngā āhuatanga o te ao Māori, i whakatinanatia e au te taukī nei, te "kaupapa Māori”. I raro i tēnei kaupapa, he tika te kōrero Māori, te whakamahi i ngā tikanga, me te whai i te mātauranga Māori. Ko te tikanga rangahau i whakamahia e au mō te tuhinga nei ko te ariā Kaupapa Māori. Nō Graham Smith (1997) te whakaaro mō te ariā Kaupapa Māori. Ko tētahi atu kaupapa rangahau i whai ahau ko te Pūrākau. Ko te whakaaro o Lee (2005), ko te Pūrākau he 
momo rangahau e hāngai atu ana ki te pakirehua ā-waha. Kua whakamahia e au te pakirehua $\bar{a}$-waha i roto i taku rangahau.

He nui ngā tuhinga e pā ana ki te tīmatanga o te kōhanga reo, arā, ko tā Hohepa (1993), ko tā Ka'ai (1990), ko tā Royal-Tangaere (1997) hoki hei tauira. E ai ki te ture kura o Aotearoa, me haere ngā tamariki kua eke ki te ono te pakeke ki tētahi kura tuatahi, kaua e noho tonu ki te kōhanga reo. Engari, i mua mai i te whakatūtanga o ngā kura kaupapa Māori, ko te kura auraki me ngā akomanga reo rua anake ngā kōwhiringa kura. He tino māharahara nō ngā mātua ki te whai akomanga, kura rānei ka tiaki i te reo o ngā raukura o te kōhanga reo. Kāore ngā kura auraki o taua wā i tino aro ki te whāngai i te reo Māori, ki te whakapūmau hoki i ngā tikanga Māori. Ki te haere ngā mokopuna o te kōhanga reo ki aua kura, kāore i roa, ka noho whakamā, ka noho mokemoke ngā tamariki nei i roto i ngā akomanga e whai ana i te reo Pākehā me ngā kaupapa Pākehā anake, $\bar{a}, \mathrm{ka}$ heke iho tā rātou whakamahi i te reo.

Nā tēnei matawaenga, i hua mai he whānau nāna i whakatū te kura kaupapa Māori tuatahi i te tau 1985 i te marae o Hoani Waititi i Tāmaki Makaurau (Smith, 1997). I whakatauira atu te kura o Hoani Waititi i tēnei kaupapa hou ki ngā whānau e rapu haere ana i ètahi rautaki hei tiaki i ā rātou raukura i puta i te kōhanga reo.

I Tāmaki Makaurau tētahi atu whānau o te kōhanga, ko Awhireinga te ingoa. He kaha wero rātou ki te kāwanatanga, i te taha ture me te taha tōrangapū, ki te whakatū i tētahi "Kaupapa Māori Language School” (ko tērā te ingoa i taua wā) mō ā rātou tamariki. I te whānau o Awhireinga a Graham Smith. I whakamārama mai ia i ō rātou tūmanako,

... to set our own model of what a kura kaupapa Māori school might be. We were arguing that the state needed to accommodate this kind of schooling. It's at that point that the kura kaupapa Māori movement became associated with the target of changing the school system to develop a state response to the kohanga reo children. (Tocker, 2014, wh. 70)

Kāore te kāwanatanga i te whakarongo ki ngā inoi ki te whakatū i tētahi kura e aro atu ana ki ngā matea o ngā raukura o te kōhanga reo. Nā tērā tūnga turi, i puaki te whakaaro o te whānau o Awhireinga ki te whawhai ki te kāwanatanga, i te taha ture me te taha tōrangapū, ki te whakatū kura kaupapa Māori.

\section{Te aromātai o te Tari Mātauranga}

I waenganui i ngā tau 1980-1990, i tutū te puehu, nā te tiwhatiwha, te pōuri me te riri o te marea e tautoko ana $\mathrm{i}$ te whakarauoratanga o te reo ki te kāwanatanga. E ai ki ngā wero, kāore te Tari Mātauranga i aro atu ki te tokomaha o ngā tamariki Māori e puta ana i ngā kura ki te ao hurihuri, kāore he tohu mātauranga, me te aha, kei raro e putu ana.

I te tau 1987, i tīmata te Pāti Reipa me te Pirimia, a David Lange, ki te hautū i te pāremata o Aotearoa. Ko Lange hoki te Minita mō te Tari Mātauranga. Nā te kaha akiaki a te marea kia whakatikahia te Tari Mātauranga, i karanga atu a David Lange ki a Brian Picot, tētahi tangata kaipakihi, kia aromātai i te whakahaeretanga o taua tari (Jones, McCulloch, Marshall, Smith \& Smith, 1990). Ā, nā Picot i ārahi tētahi rōpū matua ko "Taskforce" te ingoa, ki te wetewete i te Tari Mātauranga.

I te tau 1988, i whakatūria e te kāwanatanga ètahi Awheawhe Mahi, he rōpū mahi, hei whakatinana i ngā tohutohu a Picot. Engari kāore he rōpū e aro atu ana ki ngā hiahia o te iwi Māori kia whakatūria he kura kaupapa Māori. E ai ki a Linda Smith,

There were Working Parties on Curriculum, Special Ed, and a range of topics. On each party was a single Māori person. But it soon became clear that despite [that] they were saying that a kura kaupapa could exist, it was clear in the working party process that there was no room for them. (Tocker, 2014, wh. 84)

I kōkirikiri, i akiaki ngā tāngata Māori ki te whakatū i tētahi Awhewhe Mahi e aro atu ana ki te kaupapa o te kura kaupapa Māori. I whakamārama mai a Linda Smith, "Those of us on the [general Working Parties] said no, we're not going to participate anymore unless we can have a Working Party that basically addresses issues of the kura" (Tocker, 2014, wh. 84). Nā te tū māia o te rōpū nei mō ngā kaupapa Māori, i whakatinanatia e rātou ngā kupu o Te Aho Matua e whai ake nei,

Nō ngā Rangi Tūhāhā te wairua o te tangata. I tōna whakairatanga ka hono te wairua me te tinana o te tangata. I tērā wā tonu, ka tau tōna mauri, tōna tapu, tōna wehi, tōna mana, tōna ihi, tōna whatumanawa, tōna hinengaro, tōna auahatanga, tōna ngākau, tōna pūmanawa. (Te Aho Matua, 2008, wh. 735) 
Kātahi, ka puta te whakaaetanga ohorere o David Lange kia whakatūria tētahi Awheawhe Kaupapa Māori, arā, ko te "Kura Kaupapa Māori Working Party”. Ko John Tapiata, arā, ko te Implementation Unit Liaison Officer for Māori Education, te kaiwhakahaere o te rōpū nei (Nepe, 1991). Ko Kāterina Te Heikōkō Mataira te tiamana. Ko Pita Sharples rātou ko Aroha Sharples, ko Tuki Nepe, ko Graham Smith, ko Elizabeth Rata, ko Toni Waho, ko Cathy Dewes, ko Pem Bird, ko Rahera Shortland ngā mema o te Awheawhe Kaupapa Māori (Rata, 1989). E ai ki a Cathy Dewes,

I te hui tuatahi i karangatia e John Tapiata i tū i Okoroire, arā, i tīmata te wānanga i reira. He aha ngā hiahia, he aha ngā hē i roto i ngā kura o te wā nei, anā kua oti kē i a mātou katoa. He tino poto te wā i whakapau ia ki tērā, kātahi mātou ka aro ake ki te whakawhiti kōrero-he aha te rongoa mō ngā hē o te wā nei, me pēhea te āhua o te kura tika? (Tocker, 2014, wh. 85)

Ko tā te pūrongo “Taskforce Report”, me whakahou ngā wāhanga katoa o te Tari Mātauranga i raro i te kaupapa neoliberalism (Department of Education, 1988). Engari, he kaupapa tauaro tēnei ki ngā uara, ngā ariā me ngā tikanga Māori. Nā ngā kupu hākirikiri pēnei i ēnei: "Cultural sensitivity must play a greater part in the education system" (Department of Education, 1988, wh. 4) i mārama pai te Awheawhe Kaupapa Maori, kāore te pūrongo a Picot i āta whakaaro mō ngā wawata o te iwi Māori. Nā ngā kupu nei, "It is clear that the revival of Māori language and culture is not seen as an end in itself, but as a key to lifting the educational performance of Māori children" (Department of Education, 1988, wh. 65), i whakaatu mai te whakaaro, ko te reo Māori he waka noa iho hei whakatau i ngā tamariki Māori ki te akomanga auraki, kātahi ka huri ki te tino kaupapa, arā, ko te reo Pākehā (Smith, 1988). I pōhēhē hoki te kāwanatanga i roto kē i ngā kura auraki ngā wāhanga ako hei tiaki i ngā tamariki matatau ki te reo Māori me ngā tikanga Māori "We believe our structures will help achieve these aims" (Department of Education, 1988, wh. xiii).

Ki ngā whānau o ngā kōhanga reo, me te Awheawhe Kaupapa Maori, he tekateka noa iho èrā whakaaro. Ki te hunga Māori nei, ko te reo Māori te huarahi tika mō ā rātou tamariki me te mōhio hoki, kāore i te kaha te reo Māori i roto i ngā akomanga reo rua o ngā kura auraki i taua wā. Ki te noho ngā raukura o ngā kōhanga reo ki ēnei momo akomanga, ka mate haere tō rātou reo Māori me ngā tikanga i roto i te toru marama. Nā reira, ko te whakarauora o tō tātou reo rangatira te whāinga matua o taua Awheawhe Kaupapa Māori. I whai te rōpū Māori i ngā kupu o Te Aho Matua e whai ake nei,

He taonga te reo Māori i roto i te Tiriti o Waitangi, he reo tūturu hoki i roto i te Ture mō te Reo. Engari, kāhore he painga o te Tiriti o te Ture rānei mehemea kāore te reo i roto i te whakatumanawa, i roto i te ngākau, i roto hoki i te māngai o te iwi Māori. (Te Aho Matua, 2008, wh. 736)

Ko tētahi atu whāinga a tēnei rōpū e whawhai ana mō te kura kaupapa Māori, ko te tōkeke pāpori. Mai i te tīmatanga o ngā kura auraki, kua riro anake i ngā Pākehā ngā hua o te mātauranga, engari kua heke iho, kua mūhore ngā tamariki Māori (Smith \& Smith, 1990). Kātahi ka puta rātou i te kura ki te ao hurihuri, he tāngata kore mahi (Sharples, 1989). I hiahia te Awheawhe Kaupapa Māori ki te āta tiaki i te mauri motuhake o ngā tamariki Māori, pēnei i ngā kupu nei, "He kākano i ruia mai i Rangiātea" (Te Aho Matua, 2008, wh. 735). Nā reira, i mate nui te Awheawhe Kaupapa Māori ki te whakatū i tētahi momo kura hei whāngai i te reo Māori ki ngā tamariki kia tū māia rātou hei te wā ka puta rātou ki te ao hurihuri.

Aspirations were shaped by the frustration that our children were not going to go anywhere in mainstream. It was inevitable that we did it, otherwise our kids were going to fail-that was the story of the times. No alternative but to start our own school. (Tocker, 2014, wh. 87)

I taua wā, he kaha nō te kāwanatanga ki te aukati i ngā inoi a te whānau nei kia whakatūria he kura kaupapa Māori. Ko tētahi o ngā tauārai he whakanoho i te whānau kura kaupapa Māori i raro i te rārangi “Opt Out Clause”, he wāhi tēnei mō ngā rōpū mātāmuri, arā ko ngā hunga "whose needs are not being met adequately within the existing institutional framework will have the right to opt out of the existing school system and to create their own institutions" (Department of Education, 1988, wh. 67). He uaua rawa ki te whakatū kura Māori i raro i taua rārangi "Opt Out Clause" nā ngā tukanga manganga. I kite tōtika te whānau Māori, kāore te Taskforce a Picot i te whakapono ki te iwi Māori, ki te mana o te Māori me tana tū hei tangata whenua o Aotearoa. Anō hoki, kāore te kāwanatanga i tautoko i ngā mātāpono o te Tiriti o Waitangi.

He nui ngā piki me ngā heke engari i pākahukahu tonu te whānau ki te whakatū kura kaupapa 
Māori. Kātahi ka tae ki te marama o Tīhema, 1989, ka whakaturetia e te kāwanatanga te kura kaupapa Māori i raro i te ture "1989 Education Amendment Act, Section 155” (Nepe, 1991). Engari, he poto noa iho te harikoa mō tēnei angitu, nā te tauākī a te kāwanatanga, "Kura kaupapa Māori will be a form of designated character school" (Department of Education, 1988, wh. 1). Nā tēnei whakaahuatanga i āhei ai te kāwanatanga ki te whakahuri i ngā wāhanga rumaki reo o ngā kura auraki hei kura kaupapa Māori. E ai ki ngā mātanga nei, ki te mahi pērā, kāore e roa ka mimiti haere te kaha o te reo Māori me te mana o te kura kaupapa Māori. I whakamārama mai a Sharples,

Government taking ownership of kura watered down the criteria and caused dissension amongst the schools by designating schools to be kura when they're not what the founders decided was a kura. (Tocker, 2014, wh. 89)

I taua wā tonu, i puta te whakaaro ki te kimi rautaki anō hei whakaatu atu i te rerekētanga o ngā kura Māori i ngā wāhanga rumaki reo o roto i ngā kura auraki.

\section{Te whakawhanaketanga o Te Aho Matua}

He take tōrangapū te whakawhanaketanga o Te Aho Matua, hei whakaatu atu he kura motuhake te kura kaupapa Māori. He aukatitanga hoki $\mathrm{i}$ te wero a te kāwanatanga me tōna kaha ki te whakaiti i te tino rangatiratanga o ngā kura kaupapa Māori. E ai ki a Linda Smith, i mea atu te kāwanatanga,

... there was nothing about kura that was distinctive and therefore it should be able to exist simply as an alternative school within the way Tomorrow's Schools was expecting them to emerge. (Tocker, 2014, wh. 88)

I tētahi o ngā huihuinga, i whakawehea te Awheawhe Kura Kaupapa Māori kia rua ngā rōpū kia tutuki pai ai ngā whāinga mō te pūrongo a te kāwanatanga. I a Kāterina Te Heikōkō Mataira rātou ko Tuki Nepe, ko Cathy Dewes te mahi whakakapi i ngā tino whakaaro o te rōpū me te hanga mātāpono mō tēnei mea te kura kaupapa Māori. E ai ki a Kāterina, i reira hoki a Rahera Shortland, hei tautoko i te reo o Te Ataarangi. Nā konei i tō ai te kākano o Te Aho Matua. I whakamārama mai a Cathy Dewes,

Ka tae ki te wā kua reri mātou ki te tuhi i te pūrongo mā te kāwanatanga. I whakawehea mātou. Ka haere ake tēnei tokorua ki te aro ake ki tētahi wāhanga o te pūrongo, a wai ake ki tēnei wāhanga o te pūrongo, a wai ake ki tērā wāhanga o te pūrongo. Ka waihotia mai ko Kāterina, ko ahau, ko Tuki hei tāngata ki te kohi i ngā whakaaro katoa o te rōpū me te whakatakoto hei mātāpono, hei whāriki mō te kura hou, ana ko Te Aho Matua tērā. (Tocker, 2014, wh. 90)

Nō Kāterina Te Heikōkō Mataira ēnei whakaaro:

I remember Tuki and Cathy sitting at one end of the table and me at the other. They just wrote something and passed it to me. How we decided on those sections, I can't remember. I gave them the model, this is what we need, and broke it down to those elements, Te Ira Tangata, Te Reo, Ngā Āhuatanga Ako, and then Ngā Uaratanga [as well as Ngā Iwi and Te Ao]. We finished it on that day. A unique experience, I felt that we were guided. Absolutely sure that our tippuna were there working with us. The kōrero just flowed. I didn't stop to think, to contemplate. They passed me the piece of paper and I expanded on the thoughts that they gave to me. Mine was the hand that wrote it, but the thoughts were jointly ours, Tuki, Cathy, and me. (Tocker, 2014, wh. 90)

He āhua rite te maumaharatanga o Cathy Dewes.

I a mātou e tuhi ana, māua ko Tuki, ka tuhi i tētahi whakaaro ki runga i te pepa. Ka hoatu ki a Kāterina. Kāre ia i tino titiro ki ā māua pepa, i te tuhi noa iho ia. I taua rā i kite au i te mahi a te wairua e whakahaere ana i tana ringa. (Tocker, 2014, wh. 90)

Nā reira, e ai ki tēnei kōrero, ko Kāterina Te Heikōkō Mataira te kaiwhakawaha o Te Aho Matua. Nāna i whakapuāwai tēnei tuhinga whakahirahira. E kaha ana te aweawetia o Kāterina e Te Ataarangi me te matemate o te reo Māori. "Te Ataarangi was the major influence [and the] realization that the reo was dying" (Tocker, 2014, wh. 89). Ko tā Cathy,

Nā Kāterina, nā āna wānanga ki te wāhi ngaro i puta mai ai. Me te whakamāramatanga, he nui ngā aho o te whāriki o te mātauranga. Ko tēnei Te Aho Matua o taua whāriki. He nui noa atu, ko te Pāngarau, ko te aha rā, hei aho anō ki te whāriki o tēnei whāriki mātauranga. Anei ko "Te Aho Matua”. (Tocker, 2014, wh. 89)

Ko te reo tuatahi o Te Aho Matua, ko te reo 
Māori. He mea mīharo tēnei nā te mea i aua wā ko te nuinga o ngā kaupapa here o te mātauranga he reo Pākehā anake. I tuku atu a Kāterina mā i te tuhinga nei ki Te Tāhuhu o te Mātauranga, ki a Dr Maris O’Rourke (Chief Executive). Nā te kore mōhio o Dr Maris O’Rourke ki te reo Māori, i karanga atu ia ki tētahi atu ki te whakapākehā i ngā kupu Māori. Nā te kūaretanga o tēnei huringa ki te reo Pākehā, kātahi ko te kaha pōhēhētanga me te parahako i te tuhinga Te Aho Matua.

It was written in Māori. We had determined it would stay in Māori, and the [Chief Executive for the Ministry of Education] at that time, was adamant that she couldn't support, she didn't understand. She got Erima Henare, and then said it was a sexist document because Henare had used "he". I think it was his translation that made her feel it was a sexist document. No way would she support it. (Tocker, 2014, wh. 91)

Nā tērā mahi a te kāwanatanga, te whakatū ārai anō, i tuhia e Kāterina tētahi whakamāramatanga o Te Aho Matua i te tau 1997 (Mataira, n.d.) mō te taha o Te Rūnanga Nui o Ngā Kura Kaupapa Māori o Tāmaki Makaurau. ${ }^{1}$ He tino taumārō te kī a Kāterina mō tēnei whakamāramatanga: "I wrote an interpretation for the Minister. It should never be called a translation, it was an interpretation" (Tocker, 2014, wh. 91).

Nā te tuhinga o Te Aho Matua i tutuki pai ai te whāinga ki te whakaatu atu ki te kāwanatanga, he mana motuhake tō te kura kaupapa Māori e kawe ana i ngā ariā, ngā tikanga me ngā mātāpono rerekē rawa i ō ērā atu kura o taua wā. Ko te mea nui o Te Aho Matua ko te taha wairua.

I tuhia i mua i te take ture. Nō muri ake ko te ture. Engari ko te pūrongo ki te Kāwanatanga me tā mātou kī, "Me whakature tēnei”. Kawea e Pete tērā tohe ki te Kāwanatanga, ko John Tāpiata hoki. I mōhio mātou me whakamārama e mātou te rerekētanga o tēnei kura. Kei te tū i runga i te kaupapa Māori, ko ēnei ngā tikanga me ngā mātāpono e tika ana. Anā ko te tino rerekētanga ko te taha wairua. (Tocker, 2014, wh. 91)

Ahakoa te kaha o Pita Sharples rāua ko John Tapiata ki te akiaki i te kāwanatanga mō te taha Awhewhe Kura Kaupapa Māori ki te whakature

1 E ai ki a Rata (1991, wh. 74), i te hui ā-tau, Maehe 1991, i panoni te ingoa nei, Te Komiti o Ngā Kura Kaupapa Māori o Tāmaki Makaurau ki Te Rūnanga o Ngā Kura Kaupapa Māori o Tāmaki Makaurau. i Te Aho Matua, i noho tārewa te kaupapa nei. Tāria te wā, i te tau 1999, i whakaturetia e te kāwanatanga Te Aho Matua i runga i te kaupapa à-ture, "Education (Te Aho Matua) Amendment Act, 1999, in the repeal of the Education Act, 1989" (Tocker, 2014).

\section{Ėtahi whakamāramatanga mō Te Aho Matua}

Keingā rārangi o Te Aho Matua ngā whakamāramatanga mō te whānau o te kura kaupapa Māori, arā, me pēhea te whakauru atu i ngā uara me te mātauranga Māori ki roto i ngā akoranga o te kura me ngā hononga katoa o te whānau pēnei i te whakahaeretanga o te Poari Whakahaere Kura. E ono ngā wāhanga matua o Te Aho Matua:
1. Te Ira Tangata
2. Te Reo
3. Ngā Iwi
4. Te Ao
5. Āhuatanga Ako
6. Ngā Tino Uaratanga. (Te Aho Matua, 2008)

He mātāpono kei roto i ngā wāhanga nei hei ārahi i ngā whānau o ngā kura kaupapa Māori. Ka whakamārama atu ngā mātāpono o Te Aho Matua-ki ngā tamariki, ngā mātua, ngā kaiako, te tumuaki me te Poari Whakahaere Kura-i ngā haepapa e kawea nei i ngā tūranga katoa o te whānau. Ahakoa ko te hanga kaupapa here, ko te whakahaere rānei i te kura te mahi, ka whai tonu te Poari i Te Aho Matua. Pērā hoki i ngā kaiako. He momo mahi raranga tā ngā kaiako i te wā ka whakaurua Te Aho Matua ki roto i ngā mahere ako me te hāpainga o te marautanga.

Kāore i nui ngā tuhinga e pā ana ki te whakatipuranga o Te Aho Matua. E ai ki a Takao rātou ko Grennell, ko McGegg, ko Wehipeihana (2010), ko Te Aho Matua te tino tauira mō te whakaurutanga o ngā ariā, ngā whakapono Māori, me te reo hoki, ki ngā kura. Mā te noho pūmau ki ngā wāhanga e ono o Te Aho Matua ka mārama pai ngā iwi, he rerekē rawa te kura kaupapa Māori i te kura auraki.

Ko Nepe rāua ko Rata ētahi o te hunga whakapū i te kura kaupapa Māori. I tuhia e te tokorua nei ō rāua wheako me ō rāua whakaaro mō te orokohanga o te kura kaupapa Māori i roto i à rāua tuhinga roa mō te tohu paerua. I tana tātaritanga o te kura kaupapa Māori, i whakamārama a Nepe (1991) i te pūtake o ngā tikanga me ngā ariā i roto i ngā wāhanga o Te Aho Matua. Nā Rata (1989) tētahi kōrero e pā ana ki te hītori o Te Rūnanga o Ngā Kura Kaupapa 
Māori o Tāmaki Makaurau. Kei roto rā ko tōna whakamāramatanga o te whawhai tōrangapū me tētahi kōrero poto e pā ana ki Te Aho Matua.

I rangahau a Cooper rātou ko Arago-Kemp, ko Wylie, ko Hodgen (2004) i pēhea te whakamahi i te reo Māori o roto i ètahi o ngā kōhanga reo, ngā kura kaupapa Māori me o rātou hāpori. I roto i taua rangahau ko ētahi whakamāramatanga e pā ana ki Te Aho Matua. He mahi nui te rangahau a te rōpū o Takao mā (2010), arā, ko tā rātou uiui atu ki ngā raukura, ngā pouako, ngā tumuaki, ngā mātua, ngā whānau katoa o ètahi kura kaupapa Māori kia puta ai ō rātou whakaaro mō te angitū. I roto i te pūrongo rangahau ko ētahi tauira o ngā tikanga me te whakaaweawe o Te Aho Matua.

Nā te āhukahuka o Te Tari Arotake Mātauranga ki te kaupapa o Te Aho Matua, i mahi tahi rātou ko Te Rūnanga Nui o Ngā Kura Kaupapa Māori o Aotearoa ki te hanga i tētahi tukanga aromatawai hei aromātai i nga kura kaupapa Māori. Ko Te Aho Matua te pou tokomanawa mō te aromātai i te mahi ako i roto i ngā kura. Ko ngā wāhanga e ono o Te Aho Matua ngā paearu aromatawai (Education Review Office, 2008).

E ai ki tētahi mātanga i waihanga i Te Aho Matua, ehara Te Aho Matua i te tuhinga whakaioio, ā ka taea e ia te urutau ki ngā kura ahakoa te rerekētanga o ō rātou hapori, iwi rānei. Koinei ngā kupu tautoko a Cathy, "Ehara i te mea kua 'toka' tēnei tuhinga. Nā reira ia tau, te tikanga ia, ka whai wāhi ngā whānau katoa ki te wānanga mō Te Aho Matua ki te tīni” (Tocker, 2014, wh. 94).

Anei tētahi tauira: I te tau 1996, i tangohia e Te Rūnanga Nui te kupu "raukawa” kātahi ka whakauru atu i te kupu "raukura" kē ki te wahanga “Te Tino Uaratanga, Wāhanga 6.12” kia pēnei te haere, "Kia tū pakari, tū rangatira ia hei (raukawa) raukura mō tōna iwi" (Te Aho Matua, 2008, wh. 739). Ko te take mō tēnei huringa ko te whakaaro he tika ake te kupu "raukura" mō taua rerenga kōrero o Te Aho Matua. Ko tā Cathy anō:

I tīningia e mātou tētahi kupu engari i whakaatu atu ki a Kāterina me tana kī mai "He tika hoki. Ko te kupu ki reira ko 'raukura' ehara i a 'raukawa'." I puta mai mō roto i te ture ko te kupu "raukawa" kē ko te kōwae whakamutunga o Te Aho Matua, "kia tū rangatira te tamaiti hei raukura". Engari ko raukawa te kupu i reira, ka tīningia e mātou i tērā. (Tocker, 2014, wh. 94)

\section{Ngā whakaaro mō Te Aho Matua}

E ai ki ngā kupu o Te Aho Matua, mā te rumaki i te reo me ngā mātauranga Māori ka tū māia ngā ākonga o te kura kaupapa Māori (Te Aho Matua, 2008). He kaha rawa ō rātou tuakiri, ā ka puta ki te ao hurihuri me ō rātou mana motuhake.

Nō ngā raukura o ngā kura kaupapa Māori tuatahi i tū ki Tāmaki Makaurau, te rongo i te āhuatanga o Te Aho Matua. Ka tautoko a Mahanga Pihama i te whai i ngā mātāpono o Te Aho Matua e pā ana ki te whakahaeretanga o te kura.

Koia rā tētahi o ngā tino pūtake o Te Aho Matua, ko te whānau hei tautoko i ngā whakahaeretanga katoa o te kura. Ko ngā kaiako, te tumuaki, te poari, ko rātou e whakahaere ana i te kura. "Ko te amorangi kei mua ko te hāpai ō kei muri.” Ā, ko te whānau tērā, e hāpai ana e akiaki ana kia mōhio ai rātou i ngā whakanekeneketanga o te kura. Koia rā te ao Māori. Kei te mōhio ngā tāngata katoa, he hononga to rātou ki tērā tamaiti kotahi. Rātou katoa he kaiako ki a ia, he kaihāpai ki a ia, hei tauwhiro i a ia, hei maimai i a ia, hei tautoko i a ia. (Tocker, 2014, wh. 155)

He kōrero anō tā Tāmara mō Te Aho Matua,

Ko te tikanga o Te Aho Matua te taurahere ki wā tātou nei mātua tīpuna. Nā reira ko ngā tikanga, ko tō tātou nei reo kē te huarahi, te mātauranga hei whai. (Tocker, 2014, wh. 156)

I mea mai ngā raukura nei, ehara Te Aho Matua i te tino horopaki ako, pērā i ngā take marautanga, i ākona kētia te painga me te wairua o Te Aho Matua nā ngā tauira i whakaaturia e ngā pouako me te whānau o te kura. Nā Te Hira Paenga ēnei kupu,

We were taught Te Aho Matua indirectly. We lived it, preached it. We heard all these things, in our songs, in our haka, they were our values. (Tocker, 2014, wh. 156)

Nā ngā kupu a ngā raukura nei, ka kitea te pāpātanga o Te Aho Matua ki ō rātou whakaaro, à rātou whanonga. I noho pūmau ki Te Aho Matua, me te whakaaro koi o te tūāpapa o te kura kaupapa Māori me ngā akoranga katoa. Ki te mau pūmau ki ngā mātāpono o Te Aho Matua, kāore e kore, he pai te haere i roto i te ao. Kei Te Aho Matua te āhua o te tū, te aroha ki ngā tāngata katoa. Nā reira, i tā rātou putanga ki te ao, i whai tonu ngā raukura nei i te manaakitanga, te aroha, te āwhina,

2 Ko te hiahia o tēnei wahine kia kaua e mōhiotia ko wai ia. Nō reira, i hoatu au i tētahi ingoa anō ki a ia. 
te mahi tahi, ā, ka whakarauora hoki rātou i te reo Māori me ngā tikanga i roto i ngā rōpū kapa haka, ngā rōpu hākinakina me te wā whakangahau.

Ki raro iho nei, ka whakamārama mai a Mahanga i te wāhanga o "Te Ira Tangata”. Ki te whai i Te Aho Matua, ka āta tiaki ngā mātua i ā rātou tamariki.

Tēnei mea te tamaiti i roto i ngā āhuatanga o Te Aho Matua, ko te ira tangata te wāhanga tuatahi o Te Aho Matua. Nā wai, ka whānau mai tērā o ngā pēpē, tērā o ngā taonga, ngā kura, ngā kahurangi ki te ao mārama. Koia rā te ao i hangaia e ōna mātua, e tōna pāpā, e tōna māmā. He ao ka poipoia, he ao ka korowaitia e Te Aho Matua ka mutu e te ao Māori. (Tocker, 2014, wh. 155)

Kei te whakamahi hoki a Reikura Kahi Morgan i Te Aho Matua hei ārahi i a ia ki te whakaako i āna tamariki, ki te whakauteute i ngā tāngata katoa ahakoa te tangata, kia āwhina hoki te tuakana i te teina me te teina i te tuakana, me te mea nui hei whakakaha i āna tamariki ki te whai i te pae tawhiti.

Te Aho Matua-basic Māori tikanga. You can't go past everything that Te Aho Matua has within it, he mana anō tō te tāne, he mana anō tō te wahine, tuakana, teina; puta i te ao, whāia ngā mea e hiahia ana i tō ngākau, i tō hinengaro, hei tikitiki mō tō māhunga. I instil that within my tamariki. (Tocker, 2014, wh. 157)

Ka whakaae hoki a Chance Taylor me tana kōrero mō te aroha ki te tangata me te reo.

It's a wonderful philosophy-Te Ao Māori. It talks about principles, should be practised as a way of life. He tapu anō te tangata, ahakoa nō hea, nō wai. He tapu anō te reo. Te Aho Matua supports other iwi, other reo. (Tocker, 2014, wh. 157)

Ināianei, he tūranga pouako tō ētahi o ngā raukura nei. Kei te pouaka whakaata ētahi atu e mahi ana. Ko ètahi anō, he tāngata e whai ana i ngā tohu mātauranga i roto i ngā whare wānanga.

Kei te mōhio pai ngā raukura nei he kawenga tō rātou, he haepapa hoki e kawea nei ki ngā whānau, ki ngā hapū, ki ngā iwi hoki. Nā Mahanga ēnei kupu,

He kaitiaki mātou katoa, ngā ākonga o mua. He kaitiaki mātou mō tō tātou reo mō tō tātou momo. Ko te momo kei te kōrerohia nei e au ko te kura kaupapa Māori. Tō tātou reo, tō tātou momo, ngā kura kaupapa Māori katoa, me tō tātou korowai aroha. Ā ko Te Aho Matua tēnā. He korowai aroha, he korowai hei whakamahana, hei tiaki, hei tauwhiro i ngā tāngata katoa e hono ana ki te kura kaupapa Maōri. (Tocker, 2014, wh. 158)

Kei te whakatauira ngā raukura o ngā kura kaupapa Māori tuatahi i tū ki Tāmaki Makaurau i tēnei rārangi o Te Aho Matua, "Te piko o te māhuri, tērā te tipu o te rākau" (Te Aho Matua, 2008, wh. 736).

\section{Whakawhāititanga}

I tino kaha ngā kaihanga ki te whakapau kaha i tā rātou whawhai mō te kura kaupapa Māori i runga i te kaupapa hirahira, arā, te tū māia o ngā tamariki Māori, tā rātou noho pūmau ki te reo Māori me ōna tikanga, me tā rātou tū māia ki te ao hurihuri.

He waimarie te whānau i whakatūhia e te Pirimia Lange ngā rōpū mahi hei whakatika i ngā raruraru i waenganui i ngā wāhanga katoa o te Tari Mātauranga. I taua tatūnga, i ahei te whānau Māori ki te whakatū Awheawhe Māori e aro tōtika ana ki te kaupapa o te kura kaupapa Māori. Ko te whakapae a Graham Smith, "The only real thing that Picot Reforms have done for New Zealand in my view is actually provide the space for the kaupapa Māori development" (Tocker, 2014, wh. 82).

Whai muri i taua tohu nui, arā, te whakaturetanga o te kura kaupapa Māori, i puta ētahi atu wero e whakawehi ana i ngā whāinga o te Awheawhe Māori me te mana o te reo me ngā tikanga Māori. Nā ērā wero a te kāwanatanga, i puta ai te tuhinga o Te Aho Matua. Ko tāna, ko te ārahi i ngā whānau kura kaupapa Māori i ngā mahi katoa e hāngai ana ki te kura. Engari ko te kaupapa matua o Te Aho Matua ko te whakawhanake i ngā tamariki o te kura kaupapa Māori me ō rātou pūkenga kia āhei ai rātou ki te tū māia ki te ao Māori me te ao hurihuri, e tika ai ngā kupu nei, "Kia tū pakari, tū rangatira ia, hei raukura mō tōna iwi” (Te Aho Matua, 2008, wh. 739).

\section{Ngā tohutoro}

Cooper, G., Arago-Kemp, V., Wylie, C., Hodgen, E. (2004). Te rerenga à te pìrere. A longtidunal study of kōhanga reo and kura kaupapa Māori students. Wellington, NZ: NZCER.

Department of Education. (1988). Administering for excellence: Effective administration in education. Report of the Taskforce to Review Education Administration. Wellington, NZ: Department of Education. 
Education Review Office. (2008). A framework for review and evaluation in Te Abo Matua Kura Kaupapa Māori. Wellington, NZ: Education Review Office.

Hohepa, M. K. (1993). Preferred pedagogies and language interactions in Te Kōhanga Reo. Auckland, NZ: Research Unit for Māori Education, University of Auckland.

Jones, A., McMulloch, G., Marshall, J., Smith, L. T., \& Smith, G. (Eds.). (1990). Myths and realities: Schooling in New Zealand (pp. 21-54). Palmerston North, NZ: Dunmore Press.

Ka'ai, T. (1990). Te hiringa taketake: mai i Te Kōhanga Reo i te kura. Māori pedagogy: Te Kōhanga Reo and the transition. (Unpublished master's thesis, MPhil Education). University of Auckland, NZ.

Lee, J. B. (2005, June). Māori cultural regeneration: Pūrākau as pedagogy. Paper presented as part of a symposium "Indigenous (Māori) pedagogies: Towards community and cultural regeneration." Centre for Research in Lifelong Learning International Conference, Stirling, Scotland.

Mataira, K. (n.d.) [Collected works including: Section One: Te Abo Matua o Ngā Kura Kaupapa Māori document,1989, and Section Two: Te Abo Matua o Ngā Kura Kaupapa Māori: An interpretation of the Māori language document, ca. 1997]. Unpublished manuscript, FoEd library, The University of Auckland; the document can also be found in Official version of Te Abo Matua o ngā kura kaupapa Māori and an explanation in English, New Zealand Department of Internal Affairs, 2008, supplement to New Zealand Gazette, 32).

Nepe, T. (1991). Te Toi Huarewa Tīpuna. (Unpublished Masters thesis). The University of Auckland, NZ.
Rata, E. (1989). Māori survival and structural separateness. (Unpublished Master's thesis). Auckland University, NZ.

Royal-Tangaere, A. (1997). Learning Māori together: kōhanga reo and home. Wellington, NZ: NZCER.

Sharples, P. (1989). Kura kaupapa Māori: Recommendations for policy. In G, McCulloch (Ed.) Access: Critical perspectives on education policy. Vol. 8 no. 1: July, 1989. The Journal of the Education Department. Auckland, New Zealand: University of Auckland.

Smith, G. H. (1988). Pikau: A burden for one's back. Access 7. pp. 34-43. Auckland, New Zealand: Education Policy Group, University of Auckland.

Smith, G. H. (1997). The development of Kaupapa Māori. (Unpublished doctoral thesis, in Education). University of Auckland, New Zealand.

Smith, G. H., \& Smith, L. T. (1990). Ki te whai ao, ki te ao Kiri: Crisis and change in Māori education. In A. Jones, G. McCulloch, J. Marshall, L. Smith, \& G. Smith (Eds.), Myths and realities: Schooling in New Zealand (pp. 123-155). Palmerston North, NZ: Dunmore Press.

Takao, N., Grennell, D., McKegg, K., \& Wehipeihana, N (2010). Te piko o te māhuri. The key attributes of successful kura kaupapa Māori. Wellington, NZ: Research Division, Ministry of Education.

Te Aho Matua. (2008). Official version of Te Aho Matua o ngā kura kaupapa Māori and an explanation in English. Supplement to New Zealand Gazette, 32. Wellington, NZ: Department of Internal Affairs.

Tocker, K. (2014) Hei oranga Māori i te ao huriburi nei. Living as Māori in the world today: An account of kura kaupapa Māori. (Unpublished doctoral thesis, in Education). University of Auckland, New Zealand. 